Urologe 2020 · 59 (Suppl 2):S133-S134 https://doi.org/10.1007/s00120-020-01375-7

(c) Springer Medizin Verlag $\mathrm{GmbH}$, ein Teil von Springer Nature 2020

\section{Urologische Facharztweiterbildung im Wandel}

\author{
Ulrike Necknig ${ }^{1,2} \cdot$ Maurice Stephan Michel ${ }^{2,3}$ \\ ${ }^{1}$ Abteilung für Urologie \& Kinderurologie, Klinikum Garmisch-Partenkirchen, Garmisch-Partenkirchen, \\ Deutschland \\ ${ }^{2}$ Deutsche Gesellschaft für Urologie, Berlin/Düsseldorf, Berlin, Deutschland \\ ${ }^{3}$ Universitätsklinik für Urologie und Urochirurgie, Universitätsmedizin Mannheim, Universität Heidelberg, \\ Mannheim, Deutschland
}

Sehr geehrte Kolleginnen, sehr geehrte Kollegen,

die Urologie ist das Zukunftsfach in der Medizin. Grund dafür ist die demografisch prognostizierte Steigerung des urologischen Versorgungsbedarfs um $20 \%$, was dem über alle Fachgruppen hinweg höchsten Anstieg entspricht. Um die optimale Versorgung unserer urologischen Patienten auch zukünftig gewährleiten zu können, benötigen wir mehr denn je gut ausgebildete MedizinerInnen. Die Deutsche Gesellschaft für Urologie (DGU) ist sich dieser Mammutaufgabe bewusst und sieht sich hierfür gut vorbereitet.

Durch die Novellierung der Musterweiterbildungsordnung (MWBO) im Jahr 2018 (s. Artikel Roigas et al.) bot sich der DGU die Möglichkeit, aktiv an der Gestaltung der Weiterbildung mitzuwirken. Sie berief 2019 eine Weiterbildungskonferenz der DGU ins Leben, die sich mit der Entwicklung des fachlich empfohlenen Weiterbildungsplans (FEWP) und den konkreten Weiterbildungsinhalten befasste. Im Rahmen dessen entwickelte die Weiterbildungskonferenz in Kooperationspartnerschaft mit dem BvDU und der GeSRU als erste Fachgesellschaft in Deutschland ein DGUzertifiziertes Weiterbildungscurriculum für die Urologie gemäß der Musterweiterbildungsordnung 2018 (Fassung vom 20.09.2019). Dieses geht bereits Anfang 2021 an den Start. Einen Überblick zu den Inhalten, Rahmenbedingungen und neuen Formen der transsektoralen Zusammenarbeit geben der Leitartikel in diesem Heft sowie ein Beitrag von Westphal et al. wieder.

Eine gute Weiterbildung, die kompetente Nachwuchskräfte fördert, basiert auf verschiedenen Einflussfaktoren. Neben optimalen Ausbildungsstrukturen hängt eine gute Weiterbildung maßgeblich von einer fachlich und menschlich qualifizierten Vorbildfunktion und der Aus- bzw. Weiterbildung durch die erste und zweite Führungsebene einer Klinik ab. Die Art des Führungsstils demonstriert das Selbstverständnis, aber auch das Menschenbild des Vorgesetzten. Das Lehrer-Schüler-Verhältnis hat sich in den letzten Jahrzehnten grundlegend verändert und eine zeitgemäße Anpassung erfahren. Wie genau diese aussieht ist im Beitrag von Steffens et al. nachzulesen.

Eine optimale fachliche Weiterbildung bietet die Basis für eine spätere Berufsausübung. Angehende Urologen finden in diesem
Heft Fallbespiele, um sich auf eine bevorstehende Facharztprüfung vorzubereiten oder ihr bereits erworbenes Wissen selbst zu überprüfen. Diese Fälle stellen eine erste Auswahl dar, weitere Fälle in einem Folgeheft sind geplant.

Mit der neuen Weiterbildungsordnung wird der Kompetenzbegriff in den Fokus gerückt. Welche Fähigkeiten und Fertigkeiten sind neben der geforderten fachlichen Kompetenz für angehende Urologen wichtig? Was bedarf es, um kompetent zu sein und was kann jeder selbst dazu beitragen? Von den Berufsanfängerlnnen werden beispielsweise bereits im ersten Jahr kommunikative Kompetenzen erwartet, auf die sie mit dem Medizinstudium in der Regel nicht richtig vorbereitet sind. Stumm et al. erläutern, warum es sich lohnt, neben einem klaren Bekenntnis zu einer optimalen Ausbildung der manuellen Fertigkeiten in unserem operativ angelegten Fach auch in Kommunikationsskills zu investieren, um den eigenen Arbeitsalltag zu erleichtern und die Zufriedenheit nicht nur unserer PatientInnen, sondern auch derjenigen, die den Beruf ausüben, deutlich zu erhöhen.

Ein ganzheitlicher Ansatz für eine optimale Weiterbildung ist wichtig. Höhen und Tiefen gehören zum Leben. Auch in der urologischen Weiterbildung wird es für jeden, der sie durchläuft, zwangsläufig nicht immer nur gute Phasen geben. Krisen müssen durchlaufen werden, um Entwicklung möglich zu machen. Optimale Weiterbildung bedeutet auch hier, jungen MedizinerInnen Hilfsmittel in stressigen Zeiten zu geben, um sie resilient zu machen für die Aufgaben, die sie erfüllen wollen und sollen. Aber was ist Resilienz für Ärztinnen und Ärzte und warum kann Resilienztraining ein wichtiger Bestandteil der Weiterbildung werden? Kiesewetter et al. geben uns hierzu einen Überblick über den aktuellen Stand der Forschung.

Die Zeit der Weiterbildung fällt oftmals mit der Zeit der Familiengründung zusammen. Immer lauter wird der Ruf der Nachwuchskräfte nach einer Vereinbarkeit von Familie und Beruf. Diese Forderung ist nicht zu überhören und muss in Anbetracht des bereits existierenden Personalmangels beantwortet werden. Konzepte, diese Herausforderung zu meistern, gibt es, sie müssen nun gemeinsam umgesetzt werden (s. Necknig et al.). 


\section{Einführung zum Thema}

Liebe KollegInnen, international steht die deutsche Urologie sehr gut da - sie vereint alle urologischen Anforderungen vom Säuglingsalter bis zur Palliativmedizin, von konservativer Diagnostik über onkologische Therapien bis hin zu modernsten Operationsmethoden in einer Hand. Vor dem Hintergrund steigender Patientenzahlen und anhaltend spannender, fachlicher Weiterentwicklungen, bleibt das Fach Urologie für den Nachwuchs interessant. Um die Weiterbildung für nachkommende UrologInnen attraktiv zu gestalten, braucht es innovative Ideen und neue Wege, die gemeinsam beschritten werden.

Wir freuen uns, Ihnen mit diesem Heft interessante Aspekte zu diesem Thema bereitstellen zu können und danken allen Autoren sehr herzlich für Ihr Engagement. Wir wünschen Ihnen viel Freude beim Lesen dieser spannenden Lektüre!

\section{Korrespondenzadresse}

\section{Dr. med. Ulrike Necknig}

Abteilung für Urologie \& Kinderurologie, Klinikum Garmisch-Partenkirchen Auenstraße 6, 82467 Garmisch-Partenkirchen, Deutschland

Ulrike.Necknig@klinikum-gap.de

\section{Univ.-Prof. Dr. med. Maurice Stephan Michel}

Universitätsklinik für Urologie und Urochirurgie, Universitätsmedizin Mannheim, Universität Heidelberg

Theodor-Kutzer-Ufer 1-3, 68167 Mannheim, Deutschland

maurice-stephan.michel@umm.de

Interessenkonflikt. U. Necknig und M.S. Michel geben an, dass kein Interessenkonflikt besteht.

Ihre

Dr. Ulrike Necknig

Prof. Dr. Maurice Stephan Michel

\section{e.Medpedia: Die Online-Enzyklopädie für Ärzte}

e.Medpedia ist die neue digitale Enzyklopädie für Ärzte und ermöglicht das schnelle Nachschlagen auf Basis medizinischer Standardwerke von Springer. Die über Peer-ReviewVerfahren begutachteten Einträge werden von über 2.800 erfahrenen klinischen Experten verfasst und fortlaufend aktualisiert.

- e.Medpedia enthält alle Inhalte von über 20 etablierten Referenzwerken von Springer

- Inklusive unzähliger Abbildungen, klinischer Bilder, Tabellen und Schemata sowie Videos

- Verfasst von über 2.800 renommierten Fachärzten, gesichert durch Peer Review-Verfahren

- Komfortable Suchfunktion mit schneller Erkennung der Suchwörter

- Über 7.000 Querverlinkungen zwischen den einzelnen Einträgen

- Die bestehenden Einträge werden fortlaufend aktualisiert

- Weitere Fachgebiete werden kontinuierlich erweitert

- Mobile Nutzung über Smartphones - online und offline mit der e.Medpedia App für iOS- und Android-Geräte

Weitere Informationen:

www.springermedizin.de/emedpedia

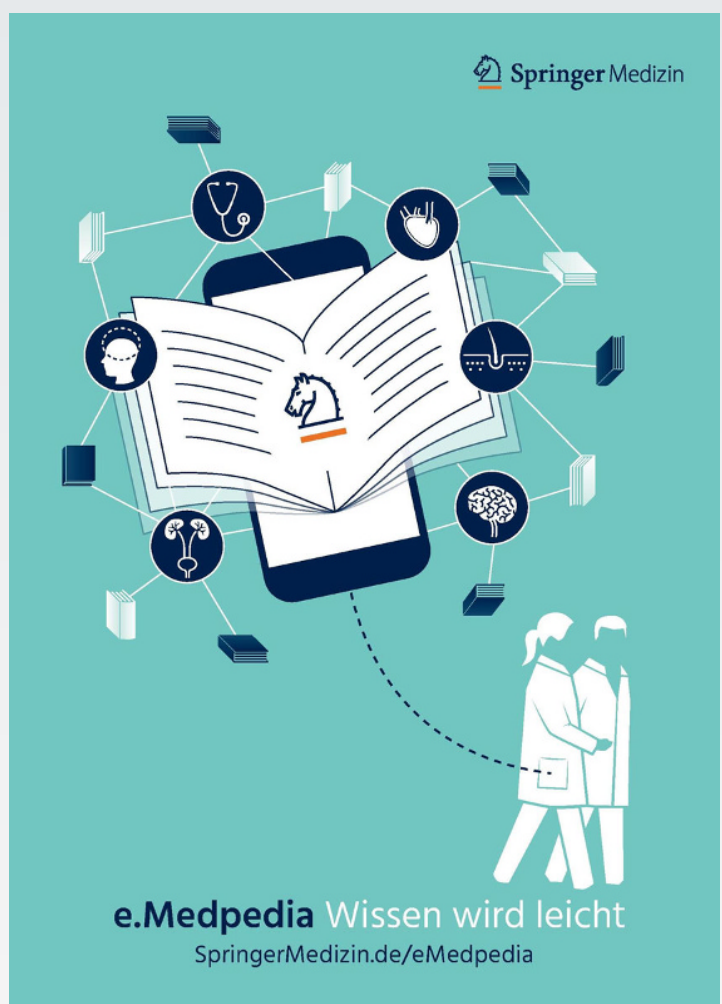

